\title{
Eigenvalue-based entropy and spectrum of bipartite digraph
}

\author{
Yan Sun ${ }^{1,2,3}$ (1) $\cdot$ Haixing Zhao ${ }^{1,3}$ \\ Received: 13 July 2021 / Accepted: 28 January 2022 / Published online: 25 February 2022 \\ (C) The Author(s) 2022
}

\begin{abstract}
Graph entropy is an important measure of the evolution and complexity of networks. Bipartite graph is a special network and an important mathematical model for system resource allocation and management. In reality, a network system usually has obvious directionality. The direction of the network, or the movement trend of the network, can be described with spectrum index. However, little research has been done on the eigenvalue-based entropy of directed bipartite network. In this study, based on the adjacency matrix, the in-degree Laplacian matrix and the in-degree signless Laplacian matrix of directed bipartite graph, we defined the eigenvalue-based entropy for the directed bipartite network. Using the eigenvalue-based entropy, we described the evolution law of the directed bipartite network structure. Aiming at the direction and bipartite feature of the directed bipartite network, we improved the generation algorithm of the undirected network. We then constructed the directed bipartite nearest-neighbor coupling network, directed bipartite small-world network, directed bipartite scale-free network, and directed bipartite random network. In the proposed model, spectrum of those directed bipartite network is used to describe the directionality and bipartite property. Moreover, eigenvalue-based entropy is empirically studied on a real-world directed movie recommendation network, in which the law of eigenvalue-base entropy is observed. That is, if eigenvalue-based entropy value of the recommendation system is large, the evolution of movie recommendation network becomes orderless. While if eigenvalue-based entropy value is small, the structural evolution of the movie recommendation network tends to be regular. The simulation experiment shows that eigenvalue-based entropy value in the real directed bipartite network is between the values of a directed bipartite small world and a scale-free network. It shows that the real directed bipartite network has the structural property of the two typical directed bipartite networks. The coexistence of the small-world phenomena and the scale-free phenomena in the real network is consistent with the evolution law of typical network models. The experimental results show that the validity and rationality of the definition of eigenvalue-based entropy, which serves as a tool in the analysis of directed bipartite networks.
\end{abstract}

Keywords Directed bipartite complex networks - Spectrum - Eigenvalue-based entropy - In-degree Laplacian matrix · In-degree signless Laplacian matrix

\section{Introduction}

Entropy is a concept that is crucial in Chemistry and Physics. It helps explain why a physical process runs in one way instead of the other, for example, why ice melts and why virus spreads out. Entropy measures disoders and represents

Haixing Zhao

h.x.zhao@163.com

1 School of Computer of Qinghai Normal University, Xining 810008, China

2 School of Computer, Qinghai Minzu University, Xining 810007, China

3 State Key Laboratory of Tibetan Intelligent Information Processing and Application, Xining, Qinghai 810001, China the state of network or system. In this work, we will use entropy to study the dynamic evolution of a bipartite graph. A bipartite graph is a complex network with a special structure. There are only two types of nodes in a bipartite graph. Each type of node set is disjoint internally, and the edges in the graph only exist between the node sets.

In real world, the networks representing fans and movies, patients and diseases, or teachers and classrooms can be abstracted into bipartite graphs. The topological structure of real networks is studied with bipartite graph model. The bipartite graph research results can be applied to venture capital investment, recommendation system, or the task assignment of teachers and classrooms in schools. The bipartite graph also has the characteristics of small world [29] and 
scale-free feature. The small-world feature refers to the shortest distance between network nodes. For example, strangers in the world can be connected through six people. The scalefree feature [10] means that the number of large-degree nodes in the network is small, while the number of small-degree nodes is large.

The current research on bipartite graphs is mainly focused on its applications. In the heterosexual contact network, males are regarded as one type of node, and females are another type of node. Liljeros et al. [17] studied the degree distribution of the vertices in the heterosexual contact network and concluded that the degree of the vertices in the heterosexual contact network follows the power-law distribution. Guler and Ergung [8] extended the priority connection mechanism in the Barabási-Albert(BA) scale-free bipartite network and established a bipartite graph with adjustable power rate distribution indices for the scale-free network evolution model of males and females. On an online shopping platform, based on the research of the bipartite network, Gharibshah [15] studied the shopping activities on social networks. This study can be applied to recommend products to consumers on the recommendation system, save online shopping time, and achieve more economic benefits [25]. In the era of big data, the bipartite graph model can also be used to protect the privacy for data release [16]. The above example applications of bipartite graph model show the importance of bipartite graph research.

However, the above mentioned research cannot fully depict all the properties of bipartite complex networks. So far, little research has been done on the dynamic evolution of bipartite network topology. To study the characteristics of the topological structure, function and evolution of the bipartite graph, a natural choice is the use of the entropy method. Entropy was first proposed in the last century when Shannon was studying information content theory [27]. In different fields, researchers proposed various definitions of entropy according to their interests [19]. In 1955, graph entropy was introduced by Rashevsky [23] for the first time. The molecules are divided into equivalence classes based on orbital symmetry from the vertex of molecular structure. Graph entropy is a very useful theoretical tool for measuring the evolution and the performance of a graph structure [6]. The graph entropy [19] of general graphs are classified into the following categories: (1) Degree-based entropy: In the real network, it is used as an index to determine the degree of node contribution. (2) Distance and degree-based entropy: Bonchev [3,12] and Trinajstic proved that the entropy is more sensitive than other classical topological single indices in mathematical chemistry. (3) Subgraph-based entropy: Konstantinova and Paleev obtained the information metric of the subgraph, which is also useful for studying the overall properties of the graph. (4) Eigenvalue-based entropy: Eigenvalue-based entropy was extended from the eigenvalue
[11] of the adjacency matrix, which was originally derived from the generalized renyi [7] entropy. Dehmer [28] proved that the use of the eigenvalue-based entropy achieved a high recognition rate for molecular structure.

In graph theory, eigenvalues can be decomposed from adjacency matrix, Laplacian matrix and signless Laplacian matrix. The eigenvalues of three matrices can be applied to different fields, each with a different role [14]. Eigenvaluebased entropy is an important branch of entropy among many others. Unfortunately, the previous studies of graph entropy were focused more on undirected graphs. A real-world directed network has more important structural properties than that of an undirected one. In the 1960s, some researchers did some initial studies on the entropy of general directed graphs such as the work done in Mowshowitz's paper [19]. For the graph entropy of a directed bipartite graph, the principle of maximum entropy [24] was used to study the contribution of two types of nodes in a bipartite graph [4]. However, there is little research on eigenvalue-based entropy of directed bipartite graphs so far. Therefore, there is a strong need to study eigenvalue-based entropy in directed bipartite graphs. In this work, we define eigenvalue-based entropy for directed bipartite graph, and calculate eigenvalue-based entropy of real directed bipartite network. The eigenvaluebased entropy of directed bipartite network is then applied to solve demanding problems of real network dynamic evolution.

The work is organized as follows: In this section, we introduce the importance of eigenvalues-based entropy in the dynamic evolution and structural properties of the directed bipartite network. In the second section, according to the directed bipartite graph theory and matrix theory, we define eigenvalue-based entropy of the adjacency matrix, in-degree Laplacian matrix and in-degree signless Laplacian matrix of the directed bipartite network. Since the eigenvalues of the three matrices in directed graphs are usually complex numbers, the corresponding eigenvalue-based entropy is also defined as the real part entropy, imaginary part entropy and modulus entropy of the eigenvalues. In the third section, we improve the construction algorithm of typical undirected networks and propose four construction algorithms of directed bipartite networks. The first part of the fourth section uses the spectrum of the in-degree signless Laplacian matrix of the directed bipartite network to do empirical analysis of the directed feature and the bipartite feature. The second part uses our definitions to calculate and analyze the nine kinds of eigenvalue-based entropy of the three matrices in the directed partite network model. Comparing with the eigenvalue-based entropy of the real directed bipartite network, we found that the eigenvalue-based entropy of the real directed bipartite graph network lies in between the directed bipartite small world and the directed bipartite scale-free network. It shows that directed bipartite network follows the evolution law of 
both the small world network and the scale-free network. This result shows that our definition of eigenvalue-based entropy are feasible in the directed bipartite network, and the research of the directed bipartite network is practically significant.

\section{Preliminaries}

An undirected bipartite graph $G=(U, V, E)$ has two types of internally disjoint subsets of vertices $U$ and $V$, where $U=$ $\{1, \cdots, s\}$ and $V=\{1, \cdots, r\}$. The edge set is denoted as $E \subseteq U \times V$, the two types of vertices associated with an edge $(r, s)$ in the bipartite graph. The $r$ and $s$ belong to the two types of vertice sets $(r \in U, s \in V)$, respectively.

Let $A(G)$ and $D(G)$ denote the adjacency matrix and degree matrix of the undirected bipartite graph $G$, respectively. The Laplacian [18] matrix is $L(G)=D(G)-A(G)$, and the signless Laplacian matrix [5] is $Q(G)=D(G)+$
The signless Laplacian matrix $Q$ of the bipartite graph $G$ is given by

$Q=\left(\begin{array}{cc}d_{r, r} & B \\ B^{T} & d_{s, s}\end{array}\right), r \neq s$

In the literature [9], the adjacency matrix and in-degree Laplace matrix is given by $A^{-}, L^{-}$, in-degree signless Laplace matrix is given by $Q^{-}$in the directed graph, respectively.

Let $G=(U, V, E)$ be a directed bipartite graph with $n$ vertices; $G$ has two types of vertex subsets. They are $U=$ $\{1,2, \cdots, r\}$ and $V=\{1,2, \cdots, s\}$. An ordered pair vertex $\left(v_{s}, v_{r}\right)$ is an arc of directed bipartite graph, i.e. the vertex $v_{s}$ walks to vertex $v_{r}(s \rightarrow r)$. The in-degree and out-degree of a vertex $r$ are denoted as $d_{r}^{-}$and $d_{r}^{+}$, respectively. The vertex $v_{r}$ in-degree sum is denoted as $\sum_{v_{s} \rightarrow v_{r}} d_{r}^{-}$. The indegree matrix and the out-degree matrix are defined as $D^{-}$ and $D^{+}$, respectively. So directed bipartite graphs, the adjacency matrix is given by

$A^{-}=\left(\begin{array}{c}0_{r, r} \\ \left(\begin{array}{ccc}1 & \text { if } r \rightarrow s \\ 0 & \text { if } r \text { is not adjacent to s }\end{array}\right)\end{array} \quad \begin{array}{c}\left.\begin{array}{cc}1 & \text { if } s \rightarrow r \\ 0 & \text { if } \mathrm{s} \text { is not adjacent to r }\end{array}\right) \\ 0_{s, s}\end{array}\right)$.

$A(G)$. The adjacency matrix $A$ of the undirected bipartite graph is as follows:
According to the formula (2), the directed bipartite graph $G$ adjacency matrix $A^{-}$is given by

$A=\left(\begin{array}{cc}0_{r, r} & \left(\begin{array}{cc}1 & \text { if } r \text { is adjacent to } s \\ 0 & \text { if } r \text { is not adjacent to } s\end{array}\right) \\ \left(\begin{array}{cc}1 & \text { if } r \text { is adjacent to } s \\ 0 & \text { if } r \text { is not adjacent to } s\end{array}\right)^{T} & 0_{s, s}\end{array}\right)$.

$T$ represents the transpose of the matrix. Let

$B=\left(\begin{array}{ll}1 & \text { if } r \text { is adjacent to } s \\ 0 & \text { if } r \text { is not adjacent to } s\end{array}\right)$.

Therefore, the adjacency matrix $A$ of the bipartite graph $G$ is simply given by

$A=\left(\begin{array}{cc}0_{r, r} & B \\ B^{T} & 0_{s, s}\end{array}\right), r \neq s$.

The Laplacian matrix $L$ of the bipartite graph $G$ is given by

$$
L=\left(\begin{array}{cc}
d_{r, r} & -B \\
-B^{T} & d_{s, s}
\end{array}\right), r \neq s .
$$

$A^{-}=\left(\begin{array}{cc}0_{r, r} & B_{s \rightarrow r} \\ B_{r \rightarrow s} & 0_{s, s}\end{array}\right)$.

In-degree Laplacian matrix $L^{-}$is given by

$L^{-}=\left(\begin{array}{cc}-1_{r, r} & B_{s \rightarrow r} \\ B_{r \rightarrow s} & -1_{s, s}\end{array}\right)$.

In-degree signless Laplacian matrix $Q^{-}$is given by

$Q^{-}=\left(\begin{array}{cc}1_{r, r} & B_{s \rightarrow r} \\ B_{r \rightarrow s} & 1_{s, s}\end{array}\right)$ 
Let $\left\{\lambda_{1}, \lambda_{2}, \cdots, \lambda_{n}\right\},\left\{\mu_{1}, \mu_{2}, \cdots, \mu_{n}\right\}$ and $\left\{q_{1}, q_{2}, \cdots, q_{n}\right\}$ be eigenvalues [13] of the adjacency matrix, in-degree Laplacian matrix and in-degree signless Laplacian matrix in directed bipartite network $G$, respectively.

Due to the asymmetry of the directed [1] bipartite network matrix, most of its eigenvalues are complex numbers, and there are positive and negative numbers among them. We propose a novel eigenvalue-based entropy relying on the adjacency matrix, in-degree Laplacian matrix and in-degree signless Laplacian matrix. Let the real part be $R e$, and the imaginary part be $I m$ in directed bipartite network. The $\left|\lambda_{j}\right|$ is the absolute value of the $j$ eigenvalue of the adjacency matrix. The $\left|\mu_{j}\right|$ is the absolute value of the $j$ eigenvalue of the in-degree Laplacian matrix. The $\left|q_{j}\right|$ is the absolute value of the $j$ eigenvalue of the in-degree signless Laplacian matrix.

We propose the definition of eigenvalue-based entropies for three matrices as follows.

\section{The eigenvalue-based entropies relying on adjacency matrix for bipartite digraph}

Definition 1 The real part entropy of the adjacency matrix is defined as

$I\left(\operatorname{Re}\left(A^{-}\right)\right)=-\sum_{j=1}^{n} \frac{\left|\operatorname{Re}\left(\lambda_{j}\right)\right|}{\sum_{k=1}^{n}\left|\operatorname{Re}\left(\lambda_{k}\right)\right|} \log \frac{\left|\operatorname{Re}\left(\lambda_{j}\right)\right|}{\sum_{k=1}^{n}\left|\operatorname{Re}\left(\lambda_{k}\right)\right|}$.

Definition 2 The imaginary part entropy of the adjacency matrix is defined as

$\operatorname{I}\left(\operatorname{Im}\left(A^{-}\right)\right)=-\sum_{j=1}^{n} \frac{\left|\operatorname{Im}\left(\lambda_{j}\right)\right|}{\sum_{k=1}^{n}\left|\operatorname{Im}\left(\lambda_{k}\right)\right|} \log \frac{\left|\operatorname{Im}\left(\lambda_{j}\right)\right|}{\sum_{k=1}^{n}\left|\operatorname{Im}\left(\lambda_{k}\right)\right|}$.

Definition 3 The modulus entropy of the adjacency matrix is defined as

$I\left(A^{-}\right)=-\sum_{j=1}^{n} \frac{\left|\lambda_{j}\right|}{\sum_{k=1}^{n}\left|\lambda_{k}\right|} \log \frac{\left|\lambda_{j}\right|}{\sum_{k=1}^{n}\left|\lambda_{k}\right|}$.

\section{The eigenvalue-based entropies relying on in-degree Laplacian matrix}

Definition 4 The real part entropy of in-degree Laplacian matrix is defined as

$I\left(\operatorname{Re}\left(L^{-}\right)\right)=-\sum_{j=1}^{n} \frac{\left|\operatorname{Re}\left(\mu_{j}\right)\right|}{\sum_{k=1}^{n}\left|\operatorname{Re}\left(\mu_{k}\right)\right|} \log \frac{\left|\operatorname{Re}\left(\mu_{j}\right)\right|}{\sum_{k=1}^{n}\left|\operatorname{Re}\left(\mu_{k}\right)\right|}$.

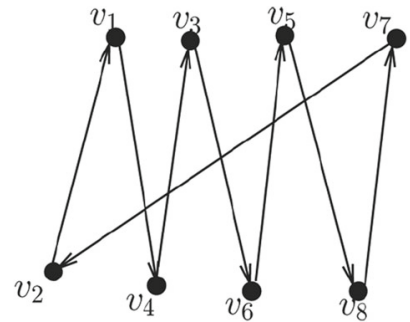

Fig. 1 An example bipartite digraph of eight vertices

Definition 5 The imaginary part entropy of in-degree Laplacian matrix is defined as

$\operatorname{I}\left(\operatorname{Im}\left(L^{-}\right)\right)=-\sum_{j=1}^{n} \frac{\left|\operatorname{Im}\left(\mu_{j}\right)\right|}{\sum_{k=1}^{n}\left|\operatorname{Im}\left(\mu_{k}\right)\right|} \log \frac{\left|\operatorname{Im}\left(\mu_{j}\right)\right|}{\sum_{k=1}^{n}\left|\operatorname{Im}\left(\mu_{k}\right)\right|}$.

Definition 6 The modulus entropy of in-degree Laplacian matrix is defined as

$I\left(L^{-}\right)=-\sum_{j=1}^{n} \frac{\left|\mu_{j}\right|}{\sum_{k=1}^{n}\left|\mu_{k}\right|} \log \frac{\left|\mu_{j}\right|}{\sum_{k=1}^{n}\left|\mu_{k}\right|}$.

\section{The eigenvalue-based entropies relying on in-degree signless Laplacian matrix}

Definition 7 The real part entropy of in-degree signless Laplacian matrix is defined as

$$
I\left(\operatorname{Re}\left(Q^{-}\right)\right)=-\sum_{j=1}^{n} \frac{\mid \operatorname{Re}\left(q_{j} \mid\right)}{\sum_{k=1}^{n}\left|\operatorname{Re}\left(q_{k}\right)\right|} \log \frac{\mid \operatorname{Re}\left(q_{j} \mid\right)}{\sum_{k=1}^{n}\left|\operatorname{Re}\left(q_{k}\right)\right|} .
$$

Definition 8 The imaginary part entropy of in-degree signless Laplacian matrix is defined as

$$
\operatorname{I}\left(\operatorname{Im}\left(Q^{-}\right)\right)=-\sum_{j=1}^{n} \frac{\left|\operatorname{Im}\left(q_{j}\right)\right|}{\sum_{k=1}^{n}\left|\operatorname{Im}\left(q_{k}\right)\right|} \log \frac{\left|\operatorname{Im}\left(q_{j}\right)\right|}{\sum_{k=1}^{n}\left|\operatorname{Im}\left(q_{k}\right)\right|} .
$$

Definition 9 The modular entropy of in-degree signless Laplacian matrix is defined as

$I\left(Q^{-}\right)=-\sum_{j=1}^{n} \frac{\left|q_{j}\right|}{\sum_{k=1}^{n}\left|q_{k}\right|} \log \frac{\left|q_{j}\right|}{\sum_{k=1}^{n}\left|q_{k}\right|}$.

Figure 1 shows an example of 8 vertices and 8 arcs in a bipartite digraph $G(U, V, E)$. We calculate the entropies of real part, imaginary part, and the modulus of the eigenvalues for the example. We obtain the adjacency matrix $A^{-}$, i.e. 
formula (19), in-degree Laplacian matrix $L^{-}$, i.e. formula (20) and in-degree signless Laplacian matrix $Q^{-}$, i.e. formula (21) from Fig. 1.

$$
\begin{aligned}
A^{-} & =\left[\begin{array}{cccccccc}
0 & 0 & 0 & 1 & 0 & 0 & 0 & 1 \\
1 & 0 & 0 & 0 & 1 & 0 & 0 & 0 \\
0 & 1 & 0 & 0 & 0 & 1 & 0 & 0 \\
0 & 0 & 1 & 0 & 0 & 0 & 1 & 0 \\
0 & 0 & 0 & 1 & 0 & 0 & 0 & 1 \\
1 & 0 & 0 & 0 & 1 & 0 & 0 & 0 \\
0 & 1 & 0 & 0 & 0 & 1 & 0 & 0 \\
0 & 0 & 1 & 0 & 0 & 0 & 1 & 0
\end{array}\right], \\
L^{-} & =\left[\begin{array}{cccccccc}
2 & 0 & 0 & -1 & 0 & 0 & 0 & -1 \\
-1 & 2 & 0 & 0 & -1 & 0 & 0 & 0 \\
0 & -1 & 2 & 0 & 0 & -1 & 0 & 0 \\
0 & 0 & -1 & 2 & 0 & 0 & -1 & 0 \\
0 & 0 & 0 & -1 & 2 & 0 & 0 & -1 \\
-1 & 0 & 0 & 0 & -1 & 2 & 0 & 0 \\
0 & -1 & 0 & 0 & 0 & -1 & 2 & 0 \\
0 & 0 & -1 & 0 & 0 & 0 & -1 & 2
\end{array}\right], \\
Q^{-} & =\left[\begin{array}{cccccccc}
2 & 0 & 0 & 1 & 0 & 0 & 0 & 1 \\
1 & 2 & 0 & 0 & 1 & 0 & 0 & 0 \\
0 & 1 & 2 & 0 & 0 & 1 & 0 & 0 \\
0 & 0 & 1 & 2 & 0 & 0 & 1 & 0 \\
0 & 0 & 0 & 1 & 2 & 0 & 0 & 1 \\
1 & 0 & 0 & 0 & 1 & 2 & 0 & 0 \\
0 & 1 & 0 & 0 & 0 & 1 & 2 & 0 \\
0 & 0 & 1 & 0 & 0 & 0 & 1 & 2
\end{array}\right] .
\end{aligned}
$$

From Fig. 1, eigenvalues are calculated via the three matrices of bipartite digraph. The calculated eigenvalues are $\lambda_{1}=-1.9999+0.0000 i, \lambda_{2}=0.0000+2.0000 i, \lambda_{3}=$ $0.0000-2.0000 i, \lambda_{4}=2.0000+0.0000 i, \lambda_{5}=0.0000+$ $0.0000 i, \lambda_{6}=0.0000+0.0000 i, \lambda_{7}=0.0000+0.0000 i, \lambda_{8}=$ $0.0000+0.0000 i$, where $\lambda_{j}, j=1,2,3, \cdots, 8$; they are the eigenvalues of adjacency matrix, where $i$ is an imaginary unit. $\lambda_{1}$ and $\lambda_{4}$ are conjugate pairs, $\lambda_{2}$ and $\lambda_{3}$ are conjugate pairs, $\lambda_{5}$ and $\lambda_{6}$ are conjugate pairs, $\lambda_{7}$ and $\lambda_{8}$ are conjugate pairs.

Similarly, the eigenvalues of in-degree Laplacian matrix are calculated as follows: $\mu_{1}=0.0000+0.0000 i, \mu_{2}=$ $2.0000+2.0000 i, \mu_{3}=2.0000-2.0000 i, \mu_{4}=3.9999+$ $0.0000 i, \mu_{5}=2.0000+0.0000 i, \mu_{6}=2.0000+0.0000 i, \mu_{7}=$ $2.0000+0.0000 i, \mu_{8}=2.0000+0.0000 i, \mu_{j}, j=$ $1,2,3, \cdots, 8$, where $i$ is an imaginary unit. $\mu_{2}$ and $\mu_{3}$ are conjugate pairs, $\mu_{5}$ and $\mu_{6}$ are conjugate pairs, $\mu_{7}$ and $\mu_{8}$ are conjugate pairs.

The eigenvalues of in-degree signless Laplacian matrix are also calculated as follows: $q_{1}=0.0000+0.0000 i, q_{2}=$ $2.0000+2.0000 i, q_{3}=2.0000-2.0000 i, q_{4}=3.9999+$ $0.0000 i, q_{5}=2.0000+0.0000 i, q_{6}=2.0000+0.0000 i, q_{7}=$ $2.0000+0.0000 i, q_{8}=2.0000+0.0000 i, q_{j}, j=$ $1,2,3, \cdots, 8$, where $i$ is an imaginary unit. $q_{2}$ and $q_{3}$ are conjugate pairs, $q_{5}$ and $q_{6}$ are conjugate pairs, $q_{7}$ and $q_{8}$ are conjugate pairs.

From above eigenvalues of the directed bipartite digraph, we observe that the eigenvalues are mostly complex numbers. Both positive and negative eigenvalues were discovered.
According to definitions 1-9 that we propose, we obtained the eigenvalue-based entropy from Fig. 1. Table 1 shows the nine eigenvalue-based entropy values of the three types of matrices from the bipartition digraph. In Table 1 , the $I\left(\operatorname{Re}\left(A^{-}\right)\right), I\left(\operatorname{Im}\left(A^{-}\right)\right)$, and $I\left(A^{-}\right)$are entropies of the real part, imaginary part, and the modulus of the adjacent matrix. $I\left(\operatorname{Re}\left(L^{-}\right)\right), I\left(\operatorname{Im}\left(L^{-}\right)\right)$, and $I\left(L^{-}\right)$are entropies of the real part, imaginary part, and the module of the in-degree Laplacian matrix. $I\left(\operatorname{Re}\left(Q^{-}\right)\right), I\left(\operatorname{Im}\left(Q^{-}\right)\right)$, and $I\left(Q^{-}\right)$are entropies of the real part, imaginary part, and the module of the in-degree signless Laplacian matrix.

As shown in Table 1, the numerical solutions of the three imaginary entropy values are the same, $\operatorname{I}\left(\operatorname{Im}\left(A^{-}\right)\right)=$ $I\left(\operatorname{Im}\left(L^{-}\right)\right)=\operatorname{I}\left(\operatorname{Im}\left(Q^{-}\right)\right)=0.6931$. Why are the values of the imaginary part entropy the same? The reasons is that Fig. 1 is a regular digraph, the in-degree direction of each vertex is same in regular digraph. Moreover, from the mathematical foundations of graph theory, in the Drago M Cvetkovi's work [5],

if a digraph is regular of a certain degree $d^{-}$, three matrices are the adjacency matrix and in-degree Laplacian matrix and in-degree signless Laplacian matrix. The relationship between three matrix is as follows. So the adjacent spectrum is,

$\left[\lambda_{1}, \lambda_{2}, \cdots, \lambda_{n}\right]$

the Laplacian spectrum is,

$\left[d^{-}-\lambda_{1}, d^{-}-\lambda_{2}, \cdots, d^{-}-\lambda_{n}\right]$

the signless Laplacian spectrum is,

$\left[d^{-}+\lambda_{1}, d^{-}+\lambda_{2}, \cdots, d^{-}+\lambda_{n}\right]$

When the eigenvalues are complex numbers, the real part of eigenvalues is a real number, and in-degree is a real number. According to (21) - (23) equation, the imaginary part entropy are equivalent for a regular digraph, i.e. the imaginary part entropy $\operatorname{I}\left(\operatorname{Im}\left(A^{-}\right)\right)=\operatorname{I}\left(\operatorname{Im}\left(L^{-}\right)\right)=\operatorname{I}\left(\operatorname{Im}\left(Q^{-}\right)\right)$. It is shown that the directional variation of the regular directed network is consistent. It shows that the imaginary part entropy has the function of describing direction of directed networks.

\section{Directed bipartition complex network model}

In recent years, researchers have conducted empirical research through the analysis of computer technology networks, cell networks, etc. Directed network [1] models have been proposed, and the characteristics and simple applications of these directed network models have been investigated. 
Table 1 The eigenvalue-based entropy of the three matrixes in the directed bipartite network in Fig. 1

\begin{tabular}{llll}
\hline Matrix & Eigenvalue-based entropy & & \\
\cline { 2 - 4 } & The real part & The imaginary part & The modular \\
\hline Adjacent matrix & $I\left(\operatorname{Re}\left(A^{-}\right)\right)=0.6931$ & $I\left(\operatorname{Im}\left(A^{-}\right)\right)=0.6931$ & $I\left(A^{-}\right)=1.3862$ \\
In-degree Laplacian matrix & $I\left(\operatorname{Re}\left(L^{-}\right)\right)=1.9061$ & $I\left(\operatorname{Im}\left(L^{-}\right)\right)=0.6931$ & $I\left(L^{-}\right)=1.9099$ \\
In-degree signless Laplacian & $I\left(\operatorname{Re}\left(Q^{-}\right)\right)=1.9061$ & $I\left(\operatorname{Im}\left(Q^{-}\right)\right)=0.6931$ & $I\left(Q^{-}\right)=1.9099$ \\
\hline
\end{tabular}

Schwartz [26] investigated the excesses of directed scale-free networks. Tadic [22] proposed a directed network model representing the www network. Ramezanpour [21] investigated a propagation process used in directed network research. Murai [20] conducted a preliminary study on the spectrum properties of a directed network.

However, the modelling of a directed bipartition network in the in-degree direction of $v_{i}$ is insufficient. The in-degree direction is from $v_{j}$ to $v_{i}$. Herein, a novel directed bipartition complex network model is proposed through algorithm improvement using a typically undirected complex network model.

\section{Directed bipartition nearest-neighbor coupling network model}

In this study, we constructed a directed bipartition nearestneighbour coupling network. In the directed bipartition network, assumption $A$ is one vertex set, and $B$ is other vertex set. The vertex of $A$ set was arbitrarily selected to connect an arc with the nearest neighbour and the direction of the arc was randomly selected. The procedures to construct the algorithm is as follows,

Step 1 Initially, assign $n$ to the node set $A$ and the node set $B$, and set a number as the $k^{\text {th }}$ nearest neighbours.

Step 2 Randomly select the $k^{\text {th }}$ nearest-neighbour node $v_{i+1+k}$ in the nodes set $A$ and random walk any node $v_{i}$ of nodes set $B$, connect the node $v_{i+1+k} \rightarrow v_{i}$ to two nodes forming an arc.

Step 3 Repeat step 2 until all $n$ different nodes are selected once.

\section{Directed bipartition small world network model}

We generate the directed bipartite small world network, by using Watts and Strogatz undirected small world network model. Construction process is as follows,

Step 1 Assign $n$ points to the node set $A$ and the node set $B$, and each point can only go to either A or B. Then initialize a directed bipartite nearest-neighbour network and randomly generate the reconnection probability $p$ and the value of $p \in(0,1)$.
Step 2 From the nearest-neighbour coupling directed bipartite network randomly select two nodes, then connect $v_{i+1+k} \rightarrow v_{i}$ to two nodes forming an arc, i.e. randomly select any node $v_{i}$ in node set $A$ random walk to $v_{i+1+k}$ of the $k^{\text {th }}$ nearest neighbour in node set $B$.

Step 3 Repeat step 2 until all $n$ different nodes are selected once.

Step 4 Generate a random number $p_{1}, p_{1} \in(0,1)$, if $p_{1} \leq$ $p$, then the arc will be randomised to reconnect; otherwise, the arc will not be reconnected.

Reconnection strategy: first, shift down the original archead in $A$ set and then randomly select another node in set $B$ as the arc-head from the unconnected nodes in set $A$ to connect with the original node.

Step 5 Until all nodes in the network are traversed.

\section{Directed bipartition scale-free complex network model}

Barabsi and Albert [2] first proposed a network model derived from the dynamic evolution of growth and priority connection mechanisms, empirically demonstrating the universal nature of a real network, where the number of nodes with large degrees is small in the network, whereas the number of nodes with small degrees is large in the network. When a directed bipartite scale-free network is constructed and illustrated with heterosexual social network, generate $m_{0}$ nodes in the female nodes set $F$ and the male nodes set $M$. First, add a new node $F_{i+1}$ to $F$ every iteration, while generate $m$ edges and use the preference probability $q=\frac{p_{j}}{\sum_{i=1}^{k} p_{j}}$, the $p_{j}$ represents the degree of the $j$ th node in the $M$ class. After that add a new node $M_{i+1}$ to $M$, use the same rules to connect edges to the $F$ class. We construct a directed scale-free complex network, where the in-degree of the node obeyed the power law distribution. The construction process of the directed scale-free network is as follows,

Step 1 Initially, assign $n$ to the nodes set $F$ and the nodes set $M$, set the number $m_{0}$ of nodes set $M$ before the network growth; randomly specify the number in nodes set $F$ of newly generated $m$ edges each time a new node $F_{i+1}$ is induced, and the network size after growth is recorded as $n$.

Step 2 Before the growth of the network (the number of nodes is $\left.m_{0}\right)$, randomly generate a number $p_{1}, p_{1} \in(0,1)$. 
When the probability is $p_{1}$, select a node $v_{i}$ of nodes set $F$ as the arc-head and another node $v_{j}$ of nodes set $M$ as the arc-end connection, which randomly connects $m_{0}$ nodes as a directed random network.

Step 3 Growth mechanism: Based on step 2), construct an algorithm program to execute $t=n-m_{0}$ time steps, add $M_{i+1}$ nodes of the nodes set $M$ in each time step from the priority of the existing nodes to priority select $m_{0}$ nodes to connect with the newly added node $M_{i+1}$, add $m$ arc each time, and calculate the cumulative in-degree connection probability $q$ (i.e. $\left.q=\sum_{i=1}^{n}\left(d_{i}^{-}\right), q \in(0,1)\right)$ of each node in the $A$ or $B$. The network does not allow repetition arcs and self-loops.

Step 4 Priority select mechanism: In step $3, m$ nodes are selected from the existing nodes in node set $F$ or $M$, and when the end of the arc is connected to the newly added node $M_{i+1}$ of node set $M$, a new node $M_{i+1}$ is added in each time step based on the probability of preference $p_{2}=\frac{d_{i}^{-}}{\sum_{i=1}^{n}\left(d_{i}^{-}\right)}=\frac{d_{i}^{-}}{q}$, $p_{2} \in(0,1)$.

Hence, the newly added nodes follow the mechanism of prioritising connections to known nodes to form a directed bipartite scale-free network.

\section{Directed bipartition random complex network model}

To construct a directed bipartite random network, we regenerate models by using undirected idea of the Erdios and Renyi [7] in this study, where the directions of the arcs are considered. Subsequently, a directed bipartite random network model is proposed. The construction process is as follows:

Step 1 Initially, the $n$ nodes are assigned to node set $A$ and node set $B$ and random connection probability $p \in(0,1)$.

Step 2 Randomly select different $p$ nodes from $A$ node set as the arc-end.

Step 3 Randomly generate a number $p_{1}, p_{1} \in(0,1)$.

Step 4 If $p_{1}>p$, select $r$ nodes in node set $B$ in step 3 as an arc-head connected by step 2 , and generate directed arcs.

Step 5 Repeat steps $1-4$ for each $v_{i}$ node, and select different nodes only once.

The arc number of the directed random network is $p\left(\begin{array}{l}n \\ r\end{array}\right)$, and the directed network does not allow repetition arcs and self-loops.

The generation model of the directed bipartite complex network is obtained through an improved algorithm of complex network typical model. The generation algorithm includes some random process. So in each running, the generated results may be different. Figure 2 shows one example of the generated directede bipartite networks. In Fig. 2, (a) is a directed bipartite nearest-neighbor coupling network, (b) is a directed bipartite small world, (c) is a directed bipar- tite scale-free network, and (d) is a directed bipartite random network. This work verifies the effectiveness of the algorithm and generates a directed bipartite network model with $n=8$ nodes.

So through Fig. 2, it is shown that the algorithm is effective and reasonable for constructing a directed bipartite network

\section{Simulation experiments}

The simulation is divided into two experiments to illustrate the structural properties and evolution of the directed bipartite network. The first experiment is the result of the spectrum of the in-degree signless Laplacian matrix of a typical directed bipartite network, which aims to illustrate the directed bipartite network. The typical bipartite complex network has the feature of directed and bipartite structure. The second experiment is the result of the three matrix eigenvaluebased entropies of directed bipartite network, which aims to illustrate the structural evolution of directed bipartite network.

\section{Spectrum of directed bipartite network}

Just as astronomers study stellar spectra to determine the composition of distant stars, one of goals of atlas theory is to derive the main properties and structure of the map from the spectrogram of the map. The research object of graph spectrum is the eigenvalue of graph. Spectrum plays a central role in our understanding of the structural characteristics of graphs. The most important structural features of the directed bipartite network are directed and bipartite. The spectrum of the directed bipartite graph indicates the structural properties of the directed bipartite network. Signless Laplacian matrix is used because researchers observed more graph properties through signless Laplacian matrix [18]. The experiment is set up as follows. The number of nodes is $n=1682$ in a bipartite network, and this value is used to compare with a real directed bipartite network. The nearest-neighbor is $k=1$ in directed bipartite nearest-neighbor coupling and small world network, the probability of small world reconnection is $p=$ 0.3 , and the reconnection probability of directed bipartite random network is $p=0.3$. Specifically, the real part of the eigenvalue of the signless Laplacian matrix $R e$ is taken as the horizontal axis and the imaginary part $I m$ as the vertical axis. The simulation results show relationship of the real part and the imaginary part of the eigenvalues in Figs. 3 and 4.

From complex function theory, the imaginary part of complex represents the direction of a vector. Observing the left subgraph in Figs. 3a, b and 4c, d, we can observe that directed bipartite nearest-neighbor network spectrum has obvious directional characteristics in Fig. $3 \mathrm{a}$, the simulation results 
Fig. 2 Generated directed bipartite network models $n=8$

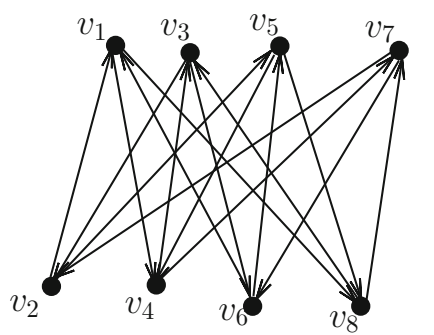

(a) Directed bipartite nearest-neighbor coupling network.

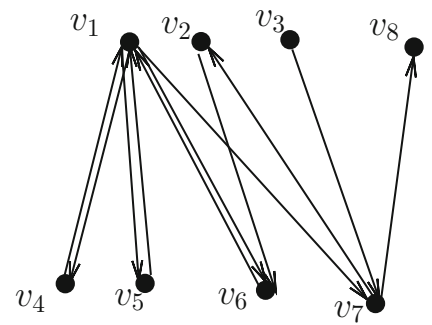

(c) Directed bipartite scale-free network.

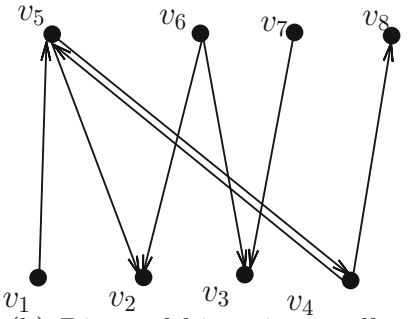

(b) Directed bipartite small world network.

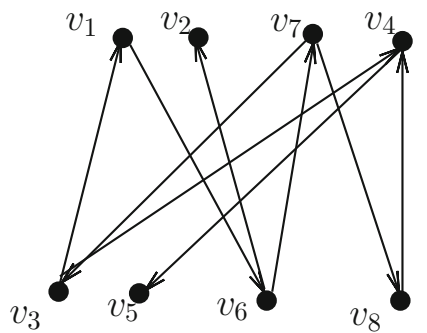

(d) Directed bipartite random network. have four petal on directed bipartite graph, the directed general graph has only one circle. The imaginary part of spectrum is two part randomly in vertical axis of complex plane, which the reconnection probability is $p=0.3$ in the directed bipartite small-world network. But the directed general graph is represented only one feature in Fig. 3b. In Fig. $4 c$ the directed bipartite scale-free network figure shows that the imaginary part direction of the two parts of the spectrum is more concentrated on the vertical axis, therefore it shows the two types of nodes follow the power law. The general directed graph is in the direction of the vertical axis, whose distribution has only a few scattered points. In Fig. 4d, both the imaginary part and the real part of the eigenvalue of the directed bipartite random network appear randomly distributed, and there are two holes in the vertical axis direction, and the general directed network does not have this phenomenon in the vertical direction. From the comparison of the directed bipartite network and the general network spectrum in Figs. 3 and 4, we obtained the obvious directed and bipartite characteristics of the typical directed bipartite network.

\section{Eigenvalue-based entropy of directed bipartite network}

Based on the typical undirected complex network generation algorithm, we improved it to a directed bipartite network model, and entropies simulation experiments on the our generation models of the directed bipartite network and the real directed bipartite network, aiming to empirically demonstrate the rationality and validity of our proposed eigenvalue-based entropy definition for directed bipartite graphs.
The real directed bipartite network is the movielens-100k dataset, which contains more than 100,000 ratings for 1682 movies by 943 users. The data set contains three tables: user table, movie information table, and rating table. The user table contains fields for user number, user gender, user age, user occupation, and user region. The user occupation includes 21 different occupations. The movie information table includes: movie number, movie name, movie release year, and movie genre. We used the movie release time and movie genre fields. The movie genre includes 19 types. The rating table contains: user number, movie number, score, and scoring time accurate to the second. It can be seen from the composition of the data set that the real data set is a directed bipartite network data set suitable for empirical study. We take the user as one part of the directed bipartite graph, and the movie is the other part of the bipartite graph, and they are disjoint internally. The users rate the movie. When using the real bipartite data set, we will ignore some fields that do not affect the real bipartite network attributes.

According to definitions 1-9, we calculated eigenvaluebased entropy of the typical directed bipartite network and of the real directed bipartite network. The experimental setting: network node $n=1682$, as shown in Fig. 5 .

In information theory, entropy is a measure of disorder in the system. The more regular the system is, the smaller the entropy is. The more disorder the system is, the greater the entropy is. According to the law of entropy, we can judge the randomness and disorder of network by calculating the entropy value. So eigenvalue-based entropy is larger in directed bipartite network model, the more dispersed the distribution of eigenvalues is, the more balanced the distribution of nodes is in the directed bipartite network. The smaller 

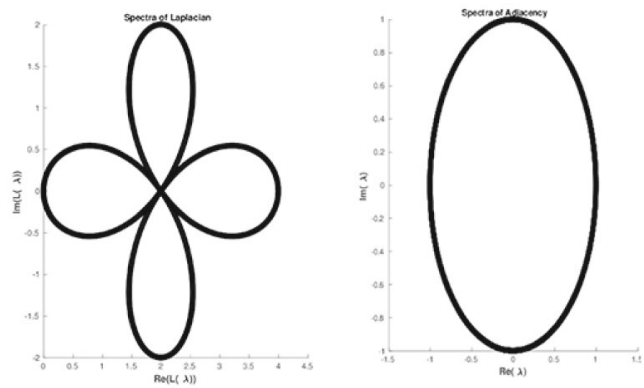

(a) Spectrum of directed bipartite vs. Nonbipartite of nearest-neighbour coupling.
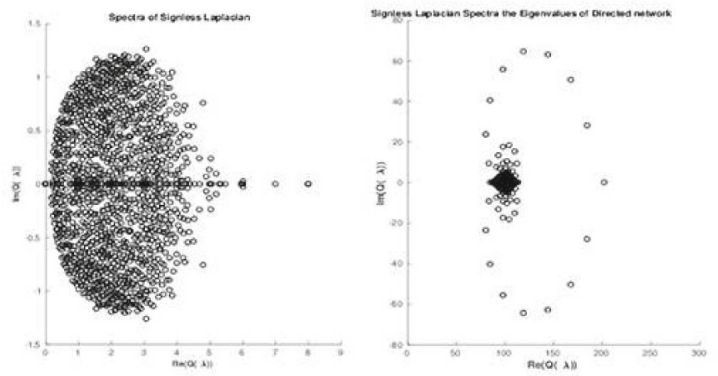

(b) Spectrum of directed bipartite vs. Nonbipartite of small world network $p=0.3$.

Fig. 3 The distribution of signless Laplacian eigenvalues of a directed bipartite vs. Non-bipartite network $(\mathrm{k}=1, \mathrm{~N}=1682)$
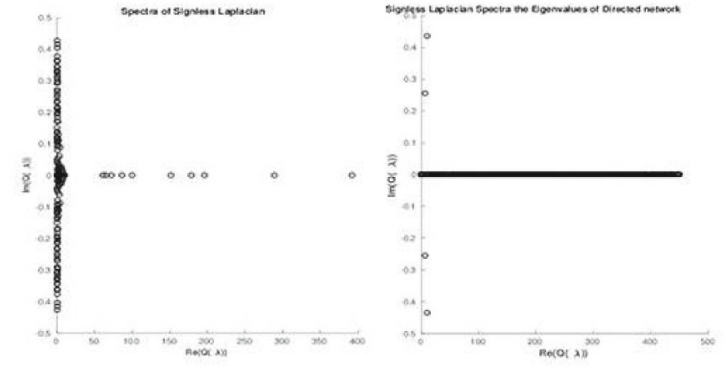

(a) Spectrum of directed bipartite vs. Nonbipartite of Scale-free network.
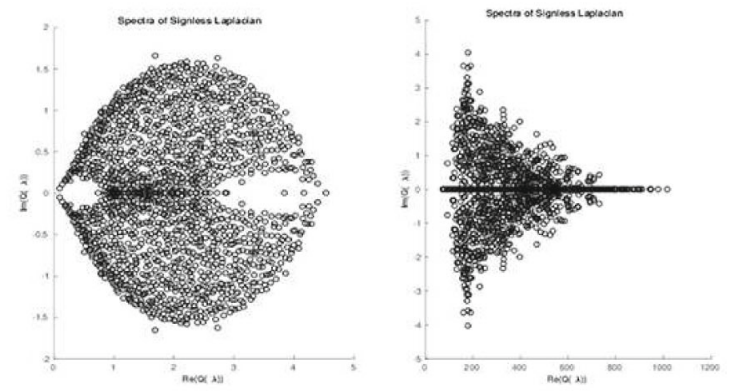

(b) Spectrum of directed bipartite vs. Nonbipartite of random network $p=0.1$.

Fig. 4 The distribution of signless Laplacian eigenvalues of a typical directed bipartite network $(N=1682)$

the eigenvalue-based entropy is, the more concentrated the distribution of the eigenvalues is, and the more unbalanced the distribution of nodes is in the directed bipartite network.

In order to study the properties of the directed bipartite network model, we assume $n=1682$ as the number of nodes in the directed network, so the nodes of the simulation experiment is consistent with nodes of the real directed bipartite network. The results of each model are the average of 100 experiments.

(1) According to the data in the BiDi-Random column in Table 2 , the nine entropy values are obtained by the directed complex random network model. Intuitively, the directions of the arcs of the directed random network must be random and multiple directional. Thus, it should be obvious that the imaginary part entropy of eigenvalue should increase. However, the imaginary part entropy value of eigenvalue of the random model shows that as the probability of node reconnection increases, the imaginary part entropy becomes smaller and smaller. The experimental results show that the directions of the arc are becoming more and more concentrated, thus the directional characteristics of the entire network are becoming more and more concentrated. This conclusion is contrary to our intuition. Next, let's describe the directed bipartite ran- dom network with a fixed reconnection probability. When the reconnection probability is $p=0.3$, the modulus entropy and real part entropy of the three matrix eigenvalues are not much different $\mathrm{I}(\operatorname{Re}(*)) I(*)>I(\operatorname{Im}(*))$, but are larger than the entropies of the imaginary part of eigenvalues of the corresponding matrix, $*$ matches $A^{-}, L^{-}$, and $Q^{-}$. It shows that the connected edges of the directed random network are becoming more and more irregular, and the distribution of directed arcs is becoming more and more balanced. However, the node connection direction of the directed bipartite random network is becoming more and more concentrated.

(2) According to the data in the BiDi-SmallWorld column in Table 2, the nine entropy values are obtained by the directed bipartite small world network model. The simulation experiment shows that the reconnection probability is $k=1, p=0.3$, and eigenvalue-based entropy of in-degree Laplacian matrix and the in-degree signless Laplacian matrix, the real part entropy and the modulus entropy of which are large, while the imaginary part entropy is small, i.e. $I\left(\operatorname{Re}\left(L^{-}\right)\right)>I\left(\operatorname{Re}\left(A^{-}\right)\right), I\left(L^{-}\right)>I\left(A^{-}\right)$ and $I\left(\operatorname{Re}\left(Q^{-}\right)\right)>I\left(\operatorname{Re}\left(A^{-}\right)\right), I\left(Q^{-}\right)>I\left(A^{-}\right)$. The imaginary part entropy is small $\operatorname{I}\left(\operatorname{Im}\left(L^{-}\right)\right)<\operatorname{I}\left(\operatorname{Im}\left(A^{-}\right)\right)$, $\operatorname{I}\left(\operatorname{Im}\left(Q^{-}\right)\right)<\operatorname{I}\left(\operatorname{Im}\left(A^{-}\right)\right)$. The results show that there is 
Table 2 Eigenvalue-based entropy of three matrix

\begin{tabular}{llllll}
\hline Entropy & \multicolumn{3}{l}{ Bipartion directed network } \\
\cline { 2 - 6 } & $\begin{array}{l}\text { Bi-Random } \\
(p=0.3)\end{array}$ & $\begin{array}{l}\text { Bi-Small world } \\
(k=1, p=0.3)\end{array}$ & $\begin{array}{l}\text { Bi-Scale free } \\
300-1682\end{array}$ & $\begin{array}{l}\text { Bi-NN coupling } \\
(k=1)\end{array}$ & $\begin{array}{l}\text { Bi-Real movie } \\
-\end{array}$ \\
\hline$I\left(\operatorname{Re}\left(A^{-}\right)\right)$ & 7.1335 & 6.9824 & 5.4964 & 7.1042 & 6.3802 \\
$I\left(\operatorname{Im}\left(A^{-}\right)\right)$ & 7.2078 & 7.0593 & 5.5809 & 7.1041 & 6.4858 \\
$I\left(A^{-}\right)$ & 7.2941 & 7.1753 & 5.6959 & 7.2830 & 6.5747 \\
$I\left(\operatorname{Re}\left(L^{-}\right)\right)$ & 7.3066 & 7.2423 & 6.2380 & 7.2768 & 6.7274 \\
$I\left(\operatorname{Im}\left(L^{-}\right)\right)$ & 7.1929 & 7.0560 & 5.2646 & 7.1041 & 5.7849 \\
$I\left(L^{-}\right)$ & 7.3272 & 7.2621 & 6.2420 & 7.3097 & 6.7274 \\
$I\left(\operatorname{Re}\left(Q^{-}\right)\right)$ & 7.3067 & 7.2426 & 6.2384 & 7.2768 & 6.7275 \\
$I\left(\operatorname{Im}\left(Q^{-}\right)\right)$ & 7.1910 & 7.0585 & 5.2700 & 7.1041 & 5.8038 \\
$I\left(Q^{-}\right)$ & 7.3275 & 7.2624 & 6.2423 & 7.3097 & 6.7275 \\
\hline
\end{tabular}

an increase and disorder towards the arc in the bipartite small-world network, and the direction of the arc tends to be concentrated. Combining the two, it shows that the changing characteristics of directed small-world networks from regular to random directed networks.

(3) According to the data in the BiDi-ScaleFree column in Table 2, the nine entropy values are obtained by the directed bipartite scale-free network model. The network node changes from 300 to 1682 , and the entropy of eigenvalue of the adjacency matrix is higher than those of the other two matrices. i.e. $I\left(\operatorname{Re}\left(A^{-}\right)\right)>I\left(\operatorname{Re}\left(L^{-}\right)\right)>$ $I\left(\operatorname{Re}\left(Q^{-}\right)\right), I\left(\operatorname{Im}\left(A^{-}\right)\right)>\operatorname{I}\left(\operatorname{Im}\left(L^{-}\right)\right)>\operatorname{I}\left(\operatorname{Im}\left(Q^{-}\right)\right)$, $\left.\left.\left.I\left(A^{-}\right)\right)>I\left(L^{-}\right)\right)>I\left(Q^{-}\right)\right)$, The network node changes from 500 to 1682 , (due to space, this dataset is not listed here) $I\left(\operatorname{Re}\left(A^{-}\right)\right)<I\left(\operatorname{Re}\left(L^{-}\right)\right)<I\left(\operatorname{Re}\left(Q^{-}\right)\right), I\left(\operatorname{Im}\left(A^{-}\right)\right)<$ $\left.\left.\left.I\left(\operatorname{Im}\left(L^{-}\right)\right)<I\left(\operatorname{Im}\left(Q^{-}\right)\right), I\left(A^{-}\right)\right)<I\left(L^{-}\right)\right)<I\left(Q^{-}\right)\right)$. The result shows that with the connection of the directed bipartite network nodes, with the increase of the optimal selection probability $p_{2}$, the directed bipartite scale-free network nodes follow the structural characteristics of the power law.

(4) According to the data in the BiDi-NN column in Table 2, nine entropy values are obtained by the directed bipartite nearest-neighbor coupling network model. For example, when the nearest-neighbor $K=1, \operatorname{I}\left(\operatorname{Im}\left(A^{-}\right)\right)=$ $I\left(\operatorname{Im}\left(L^{-}\right)\right)=\operatorname{I}\left(\operatorname{Im}\left(Q^{-}\right)\right)=\mathbf{7 . 1 0 4 1}$, the entropy numerical value of imaginary part is equal in the three types of matrixes of the directed bipartite nearest-neighbor coupling network. The result verifies that the nodes directions are consistent in the directed nearest-neighbor coupling network. The simulation of the directed nearest-neighbor network show consistent results with the theoretical analysis in the directed network.

The simulation experiment shows that the definition of eigenvalue-based entropy can effectively quantify the structural evolution characteristics of the directed bipartite network.
In the subgraph (a) of Fig. 5, the horizontal axis is the entropy of real part $I(R e)$ of the adjacency matrix $A$, the entropy of the imaginary part $I(I \mathrm{~m})$ of the adjacency matrix $A$, and entropy modulus $I$ of the adjacency matrix. The vertical axis is represented entropy value by the five networks, and they are directed bipartite random, directed bipartite small world, directed bipartite scale free, directed bipartite nearest-neighbor coupling network, directed bipartite real movie scoring network.

The horizontal axis of the subgraph (b) of Fig. 5 is the entropy of real part $I(R e)$, the entropy of imaginary part $I(I m)$, and entropy modulus $I$ of the in-degree Laplacian matrix $L$, The vertical axis is entropy value from the five networks.

The horizontal axis of the subgraph (c) of Fig. 5 is the entropy of real part $I(R e)$, the entropy of imaginary part $I(I m)$ and modulus $I$ of the in-degree signless Laplacian matrix $Q$, the vertical axis is entropy value the five networks.

Observing the nine entropy values in the Bi Di-Movie column in Fig. 5, eigenvalue-based entropy of the real directed bipartite movie scoring network is middle the directed bipartite small world and the directed bipartite scalefree network. Therefore, the real movie bipartite network has the structural characteristics of a directed small world and a directed scale-free network.

\section{Conclusion}

In our work, aiming at property of spectrum and structure evolution in the directional bipartite network, by modeling the directed bipartite complex network. The spectrum shows static properties, and eigenvalue-based entropy represents dynamic feature of $\mathrm{d}$ irectional bipartite network. We constructed the directed bipartite nearest-neighbor coupling network, the directed bipartite small-world network, the directed bipartite scale-free network, and the directed 


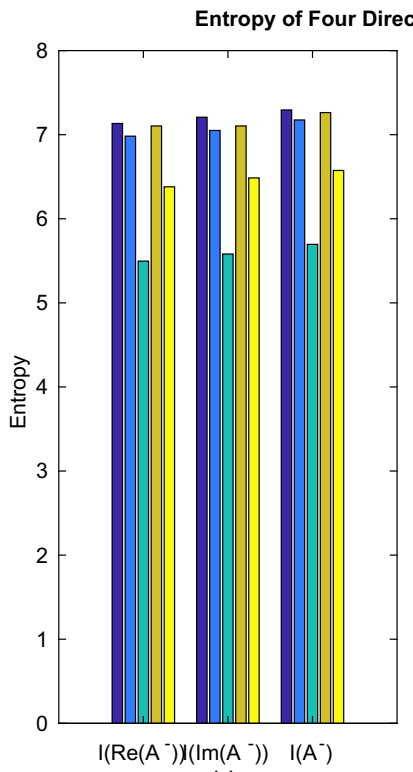

(a)

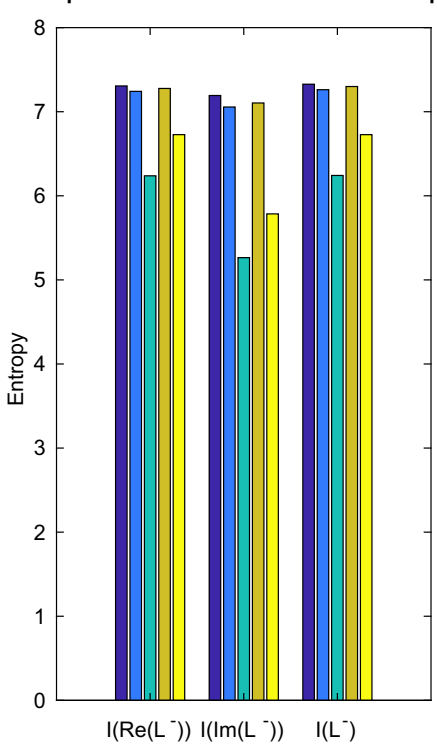

(b)

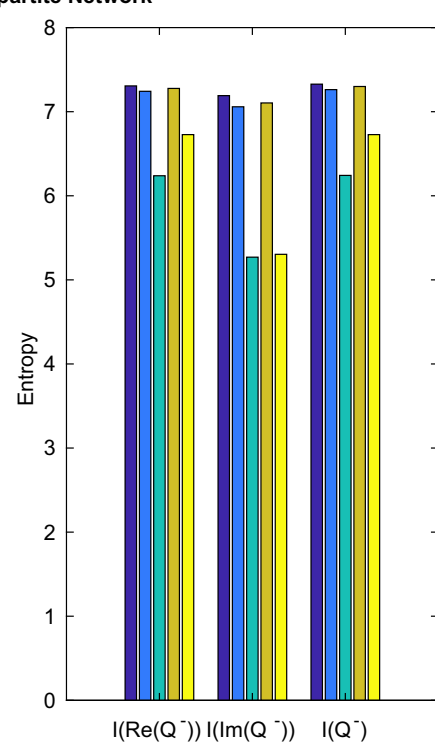

(c)

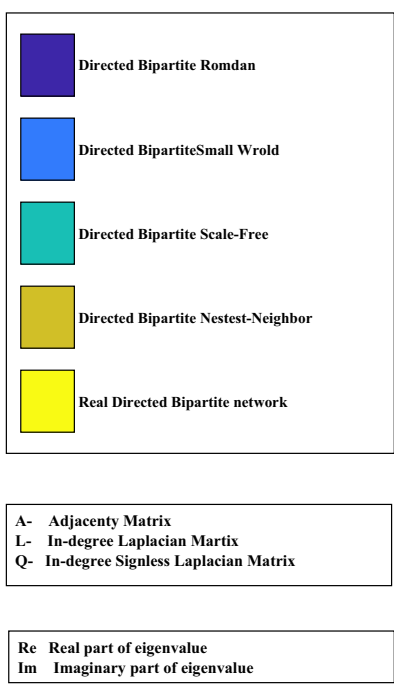

Im Imaginary part of eigenvalu

Fig. 5 The three matrix eigenvalue-based entropy of constructed and real directed bipartite network

bipartite random network. Eigenvalue-based entropy of three kinds of matrixes has been studied. Since the three kinds of matrices of the directed bipartite complex network are asymmetric matrices, and their eigenvalue are mostly complex numbers, this work propose the entropy definition of the real part, imaginary part and modulus of eigenvalue via the adjacency matrix, the in-degree Laplacian matrix and the in-degree signless Laplacian matrix in the directed bipartite network.

The simulation experiment demonstrated the characteristics of the nine eigenvalue-based entropy of the three matrices in a real network and the constructed directed bipartite networks. First, the relationship between the real and imaginary parts of the eigenvalue in a typical directed bipartite network, the characteristics of the directed bipartite are directed and bipartite. Second, entropies of the imaginary part of in three type matrices are calculate, the directional characteristics of the arcs is obtained in the directed bipartite network, i.e. the entropy of imaginary part are equal from the directed bipartite nearest-neighbour coupling network. Thus, the imaginary part of eigenvalue-based entropy effectively describes the directional characteristics of the directed bipartite network. Third, the simulation results of a realistic directed bipartite complex network(database of movie scoring) show that the structural characteristics of the real directed bipartite network are between the directed bipartite scale-free and small-world networks. The results of the structural characteristics in the directed bipartite network can be applied to the real directed bipartite network.
Acknowledgements We thank the editors and the anonymous reviewers for their professional and valuable suggestions. Research supported the Science and Technology Plan of Qinghai Province, China (Grant No. 2019-ZJ-7012). The Tibetan Information Processing and Machine Translation Key Laboratory of Qinghai Province (No.2020-ZJ-Y05), and the Key Laboratory of Tibetan Information Processing Ministry of Education and Tibetan Information Processing Engineering Technology and Research Center of Qinghai Province.

\section{Declarations}

Conflict of interest The authors declare that they have no conflict of interest.

Open Access This article is licensed under a Creative Commons Attribution 4.0 International License, which permits use, sharing, adaptation, distribution and reproduction in any medium or format, as long as you give appropriate credit to the original author(s) and the source, provide a link to the Creative Commons licence, and indicate if changes were made. The images or other third party material in this article are included in the article's Creative Commons licence, unless indicated otherwise in a credit line to the material. If material is not included in the article's Creative Commons licence and your intended use is not permitted by statutory regulation or exceeds the permitted use, you will need to obtain permission directly from the copyright holder. To view a copy of this licence, visit http://creativecomm ons.org/licenses/by/4.0/.

\section{References}

1. Ayyaswamy S, Balachandran S, Gutman I (2011) Upper bound for the energy of strongly connected digraphs. Appl Anal Discret Math $5(1): 37-45$ 
2. Barabasi AL, Albert R (1999) Emergence of scaling in random networks. Science 286(5439):509-512

3. Bonchev D, Trinajsti N (1977) Information theory, distance matrix, and molecular branching. Chem Phys 67(10):4517-4533

4. Burgos E, Ceva H, Hernandez L, Perazzo RP, Devoto M, Medan D (2008) Two classes of bipartite networks: nested biological and social systems. Phys Rev E Stat Nonlinear Softw Matter Phys 78(4 Pt 2):046113

5. Cvetkovi DM, Doob M, Sachs H (1980) Spectra of graphs,theory and application. Spectra Graphs Theory Appl

6. Ebel H, Mielsch LI, Bornholdt S (2002) Scale-free topology of e-mail networks. Phys Rev E 66(3Pt2A):035103

7. Erdios P, Renyi A (1959) On random graphs. Publicationes Mathematicea 6:290-297

8. Ergung (2002) Human sexual contact network as a bipartite graph. Phyical A 308(1):483-488

9. F, F.K (2003) Some properties of directed graphs. J Ningbo Univ Nat Sci Edn 3:228-231

10. Faloutsos M, Faloutsos P, Faloutsos C (1999) On power-law relationships of the internet topology. Proc Acm Sigcomm 29(4):251262

11. Farkas IJ, Dernyi I, Barabsi A, Vicsek T (2001) Spectra of "realworld" graphs: Beyond the semicircle law. Phys Rev E Stat Nonlinear Softw Matter Phys 64(2):026704

12. Ivanciuc O, Balaban TS, Balaban AT (1993) Chemical graphs with degenerate topological indices based on information on distances. J Math Chem 14(1):21-33

13. Ivanciuc O, Ivanciuc T, Diudea MV (2010) Cheminform abstract: Polynomials and spectra of molecular graphs. Cheminform 7(1):41-67

14. Ivanciuc O, Ivanciuc T, Klein DJ, Seitz WA, Balaban AT (2001) Wiener index extension by counting even/odd graph distances. J Chem Inf Comput 41(3):536-549

15. Javari A, Gharibshah J, Jalili M (2014) Recommender systems based on collaborative filtering and resource allocation. Soc Netw Anal Min 4(1):234

16. Lihui L, Shiguang J, Hu J (2010) Privacy-preserving data publishing using bipartite graph. Appl Res Comput 27(11):4303-4308

17. Liljeros F, Edling C, Amaral R, Nunes LA, Stanley EE, Aberg Y (2001) The web of human sexual contacts. Nature

18. M, B, P, N (2001) Laplacian eigenmaps and spectral techniques for embedding and clustering. Adv Neural Inf Process Syst 14(6):585591
19. Mowshowitz A (1968) Entropy and the complexity of graphs: Ii. the information content of digraphs and infinite graphs. Bull Math Biol 30(2):225-240

20. Murai T (2003) Spectral analysis of directed complex networks. The Physical Society of Japan (JPS)

21. Ramezanpour A, Karimipour V (2002) Simple models of small world networks with directed links. Phys Rev E Stat Nonlinear Soft Matter Phys 66(3):036128

22. Randic M, Muller WR, Knop JV (1997) The characteristic polynomial as a structure discriminator. J Chem Inf Comput Sci 28(6): 1072-1077

23. Rashevsky N (1955) Life, information theory, and topology. Bull Math Biophys 17(3):229-235

24. Rodder W, Dellnitz A, Kulmann F, Litzinger S, Reucher E (2019) Bipartite structures in social networks: Traditional versus entropydriven analyses. Entropy (Basel) 21(3)

25. S, G (2011) The microstructure of the money market before and after the financial crisis, a network perspective. CEIS Tor Vergata, Research Paper Series 9(1):181

26. Schwartz N, Cohen R, Ben-Avraham D, Barabsi A, Havlin S (2002) Percolation in directed scale-free networks. Physical Review E Statistical Nonlinear Soft Matter Physics 66(2):015104

27. Shannon A (1948) mathematical theory of communication. Math Theory Commun

28. Sivakumar L, Dehmer M, Varmuza K (2012) Uniquely discriminating molecular structures using novel eigenvalue-based descriptors. MATCH Commun Math Comput Chem 67(1):147-172

29. Watts DJ, Strogatz SH (1998) Collective dynamics of "smallworld" networks. Nature 393:440-442

Publisher's Note Springer Nature remains neutral with regard to jurisdictional claims in published maps and institutional affiliations. 